\title{
Intervista ad Adamasco Cupisti
}

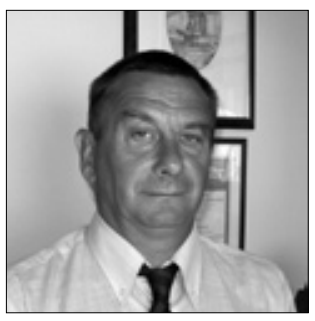

Adamasco Cupisti, MD, PhD

Divisione di Nefrologia

Dipartimento di Medicina Interna

Università di Pisa

acupisti@med.unipi.it

Nel Centro ove presti attività pratichi il dosaggio della $25(\mathrm{OH}) \mathrm{D}$ e/o della $1,25(\mathrm{OH})_{2} \mathrm{D} 3$ ?

Se sì, in quale tipo di paziente e in quale percentuale? Se no, perché ritieni di non doverla praticare?

Nel nostro Centro è disponibile il dosaggio sia della $25(\mathrm{OH})$ D sia della 1,25 $(\mathrm{OH}) \mathrm{D}$. Nella routine clinica, richiediamo il dosaggio della $25(\mathrm{OH}) \mathrm{D}$ in una elevata percentuale di pazienti con $\mathrm{CKD}$. Invece, il dosaggio della $1,25(\mathrm{OH}) \mathrm{D}$ è praticato solo in casi particolari, quali ad esempio condizioni di ipercalcemia o di bassi livelli di PTH.

Applichi la correzione di $25(\mathrm{OH}) D$ in caso di carenza? Perché, con quale protocollo e in quale tipo di paziente?

Qualora si riscontri una deficienza di VitD (livelli circolanti di $25(\mathrm{OH}) \mathrm{D}<30 \mathrm{ng} / \mathrm{mL})$, consigliamo la correzione con colecalciferolo (ad esempio, Di Base: 15-20 gocce alla settimana), ricontrollando nel tempo i livelli di $25(\mathrm{OH}) \mathrm{D}$. Lo scopo è di ottenere i potenziali effetti benefici riportati sui livelli di PTH, sul sistema immunitario e sulla morbidità e mortalità cardiovascolare.

Nel Centro ove presti attività in quale percentuale o in quali casi clinici particolari pratichi la biopsia ossea?

Nel nostro Centro, la biopsia ossea non viene praticata.

Nel Centro ove presti attività in quale percentuale $e$ in quali stadi di CKD usi indagini strumentali per la ricerca delle calcificazioni vascolari? Quali indagini utilizzi?

Non eseguiamo l'analisi sistematica delle calcificazioni vascolari nei pazienti con CKD. La ricerca delle calcifica- zioni vascolari viene effettuata nei candidati al trapianto renale, mediante Rx addome e arti inferiori. L'ecocardiogramma viene invece effettuato sistematicamente.

Nel Centro ove presti attività qual è la percentuale di pazienti in trattamento sostitutivo che resta nel range di normalità (secondo le linee guida SIN) per Ca, P e PTH?

Il $70 \%$ dei pazienti ha una fosforemia $<5,5 \mathrm{mg} / \mathrm{dL}$, il $18 \%$ una calcemia corretta $>9,6 \mathrm{mg} / \mathrm{dL}$, e il $15 \%$ un prodotto calcio ${ }^{\star}$ fosforo $>55 \mathrm{mg}^{2} / \mathrm{dL}^{2}$. Per quanto riguarda i livelli di iPTH: $>300 \mathrm{pg} / \mathrm{mL}$ nel $36 \%$ dei pazienti $(12 \%>450 \mathrm{pg} /$ $\mathrm{mL}), \mathrm{e}<150 \mathrm{pg} / \mathrm{mL}$ nel $30 \%$.

Nel Centro ove presti attività qual è la percentuale dei vari chelanti usati per ricercare il controllo del Ca-P nei pazienti in trattamento sostitutivo (anche in off-label treatment)?

Sali di calcio (da 400 a $1600 \mathrm{mg}$ di calcio elemento) vengono usati in quasi tutti i pazienti (84\%). A oggi, il sevelamer è impiegato nel $22 \%$ dei pazienti, il carbonato di Lantanio nel 9\%, mentre nessun paziente assume alluminio.

Nel Centro ove presti attività qual è la percentuale di pazienti in CKD 3-5 sottoposta a dieta ipoproteica per il controllo della iperfosforemia?

In tutti i pazienti, a partire dallo stadio CKD III è consigliata una "normalizzazione" dell'apporto di proteine, con informazione ed educazione al controllo dell'apporto di fosforo e sodio. Negli stadi CKD IV-V, a tutti i pazienti è consigliata una dieta ipoproteica $(0,6 \mathrm{~g} / \mathrm{kg} / \mathrm{die} o$ $0,3 \mathrm{~g} / \mathrm{kg} /$ die supplementata con amino acidi essenziali e chetoanaloghi), a meno che non esistano controindicazioni cliniche o socio-economiche, o mancanza di consenso o di aderenza. Negli stadi CKD IV-V la percentuale effettiva di pazienti in dieta ipoproteica-ipofosforica, con o senza l'impiego dei prodotti aproteici, è intorno al 70\%, con un grado di buona aderenza nel $50 \%$ dei pazienti trattati. A questi livelli di funzione renale residua si informa il paziente anche sulla possibilità di una dieta fortemen- 
te ipoproteica e ipofosforica supplementata con amino acidi essenziali e chetoanaloghi, che viene attuata solo in pazienti selezionati, motivati e sotto stretto controllo clinico e nutrizionale.

Nello stadio 5D, si forniscono indicazioni generali al paziente per limitare l'apporto di fosforo, sodio e potassio pur mantenendo quello di proteine. Questo avviene tramite counselling dietetico-nutrizionale.

Nel Centro ove presti attività quale percentuale di pazienti in trattamento sostitutivo che assumono vit-D o analoghi, calciomimetico, o entrambi?

Nel nostro Centro, a oggi, la percentuale di pazienti che assumono vitD o analoghi è del $48 \%$, e il calciomimetico è assunto dal $24 \%$ dei pazienti.

Nel Centro ove presti attività qual è la percentuale di pazienti monitorati per CKD-MBD stadio 3-5 e quali marker biologici usi a tal fine?

Calcemia, fosforemia, fosfatasi alcalina, PTH e bicarbonatemia sono regolarmente richiesti per monitorare la CKD-MBD e per permettere i necessari aggiustamenti della terapia.

Le calcificazioni vascolari - evento davvero comune nel paziente con CKD - sono associate a un elevato rischio di mortalità in dialisi ma non esistono studi d'intervento che dimostrino chiaramente che la loro riduzione migliori la sopravvivenza. Come va interpretato tale apparente paradosso?

Le evidenze che mettono in relazione l'elevato rischio di mortalità in dialisi con il grado di calcificazioni vascolari derivano da studi di popolazione e quindi, per quanto suggestivi, non implicano un rapporto causa-effetto esclusivo. Del resto l'estesa multifattorialità del danno vascolare con o senza calcificazione nei pazienti in dialisi è ben noto. Questo, unito alle molte comorbidità che caratterizzano il paziente in dialisi e alla presumibile lentezza del processo di calcificazione (e della sua potenziale reversibilità), complica drammaticamente la possibilità di dimostrare in trial clinici il rapporto tra riduzione delle calcificazioni e aumentata sopravvivenza. Inoltre a oggi, le metodiche di imaging adatte per questo studio sono relativamente grossolane o troppo complesse e costose.

PTH e CKD-MBD: quanto artefice o quanto vittima? Ad esempio la carenza di vitamina $D$ (o meglio la riduzione della attivazione dei suoi recettori nucleari) è sicuramen- te un evento che precede l'iperparatiroidismo secondario. Quale di questi due giocatori è in "fuori gioco" per lo sviluppo della patologia ossea dell'uremico e a quale dei due - se possibile - si può imputare un predominio sullo sviluppo di CKD-MBD?

La carenza di vitamina D (o meglio la riduzione della attivazione dei suoi recettori nucleari) è uno degli eventi che precedono l'iperparatiroidismo secondario, ma la tendenza alla ritenzione di fosforo, dovuta a un carico dietetico di fosforo eccessivo per la funzione renale residua, rappresenta l'aspetto centrale della fisiopatologia dell'iperparatiroidismo secondario alla CKD. Del resto è noto che il carico di fosforo contribuisce alla riduzione della sintesi di calcitriolo, e la dieta ipofosforica stimola l'1 $\alpha$-idrossilasi, almeno nelle prime fasi della CKD. Le nuove acquisizioni di fisiopatologia identificano nella fosfotonina FGF-23 l'elemento di congiunzione fra il carico dietetico di fosforo e i livelli di calcitriolo e quindi di PTH. La fosforemia, nella norma fino agli stadi terminali della $\mathrm{CKD}$, non può essere considerata un indicatore affidabile di ritenzione di fosforo né un elemento per guidare la terapia. Servirebbe un marker di bilancio del fosforo, e in questo senso si hanno promettenti novità dal FGF-23. Quindi, senza nulla togliere agli altri attori, maggiore attenzione dovrebbe essere posta al controllo dell'apporto di fosforo nella $\mathrm{CKD}$.

Dopo oltre un trentennio di osservazioni, esperienze, studi $e$ ricerche, la fisiopatologia delliperpararatiroidismo secondario non è ancora del tutto chiarita. Calcio, fosforo, recettori della vit-D o recettori sensibili del calcio, ormoni fosfatotropi e forse l'esistenza di un recettore cellulare del fosforo, sono tutti "in carriera" assieme al primo attore il PTH (almeno nella cronologia storica): si è in grado di prevedere un ordine e chiarimento delle cose o dobbiamo ancora e solo predire?

La patogenesi multifattoriale della CKD-MBD è ben evidente. Fosforemia, calcemia, calcitriolo e PTH hanno ciascuno un ruolo molto importante, ma la tendenza alla ritenzione di fosforo rappresenta il primum movens. Infatti, il fosforo è in grado di inibire l'1 $\alpha$ idrossilasi renale direttamente, e indirettamente tramite l'incremento del FGF-23: la conseguente riduzione dei livelli di calcitriolo stimola la sintesi e secrezione del PTH. Inoltre esiste evidenza che l'incremento della fosforemia, indipendentemente dalle modificazioni di calcemia e calcitriolo, stimola direttamente sintesi di PTH e, quello che è più grave, l'iperplasia delle paratiroidi. La recente definizione delle azioni del FGF-23 nell'ambito del metabolismo 
calcio-fosforo ha reso ancora più complessa l'interazione fra i succitati attori ma ha contribuito a confermare il ruolo chiave e precoce della ritenzione di fosforo sui meccanismi della CKD-MBD. Infatti, l'FGF-23 aumenta nelle fasi precoci della CKD e soprattutto in risposta a un carico dietetico di fosforo: forse questo rappresenta uno dei tasselli mancanti in grado di giustificare il ruolo della fosforo-ritenzione nel determinismo della CKD-MBD e contribuisce a giustificare il controllo del fosforo dietetico e la riduzione dell'assorbimento intestinale netto di fosforo fin dagli stadi molto precoci della CKD.

Perché supplementare con calcifediolo e calcitriolo $i$ pazienti in CKD 3-5, con carenza di 25(OH)D?

La carenza di vitamina $\mathrm{D}$ è definibile per livelli di calcifediolo circolanti $<30 \mathrm{ng} / \mathrm{mL}$, che diviene importante per livelli $<15 \mathrm{ng} / \mathrm{mL}$. Questa condizione è molto frequente nei pazienti con CKD e si aggrava con la progressione dell'insufficienza renale, ma si riscontra in percentuali molto alte anche nella popolazione generale. Benché la correzione della carenza di vit.D non si traduca necessariamente in un aumento dei livelli di calcitriolo (dato il più o meno grave deficit di $1 \alpha$-idrossilasi renale), essa può avere effetti favorevoli sul PTH, sul sistema immunitario e sulla patologia cardiovascolare.

\section{Quale ruolo affidi alle norme dietetiche nel controllo della fosforemia nel paziente in emodialisi?}

Nel paziente in dialisi, la dieta da sola non è in grado di controllare il bilancio del fosforo perché l'entità della riduzione dell'apporto di fosforo risulta limitata sia per il conflitto con l'aumentato fabbisogno proteico sia per comprensibili problemi di aderenza alle restrizioni dietetiche. Tuttavia, l'assenza di educazione alimentare o di norme dietetiche invariabilmente aggrava la positività del bilancio del fosforo e limita fortemente l'efficacia degli altri provvedimenti terapeutici. Al contrario, la limitazione dell'apporto dietetico di fosforo è facilmente ottenibile e necessaria per rendere più efficace l'effetto dei chelanti intestinali del fosforo, con evidente vantaggio in termini di compliance e di costi, e per concedere più spazio all'uso del calcio e del calcitriolo.
Quali strumenti ritieni utili per limitare l'apporto dietetico di fosforo nel paziente in emodialisi, senza limitare l'introduzione di proteine?

Un approccio semplice ed efficace per ridurre il carico dietetico di fosforo, senza ridurre quello di proteine, consiste nell'istruire i pazienti a non assumere alimenti ad alto contenuto di fosforo (ad esempio, formaggi, tuorlo d'uovo, frutta secca ecc.), a non acquistare alimenti contenenti additivi a base di polifosfati, e a preferire la bollitura ad altri metodi di cottura.

\section{Commento conclusivo}

Le conclusioni non possono non essere sovrapponibili a quelle dei colleghi che mi hanno preceduto. Ad integrazione, la mia opinione è che dovrebbe essere praticata maggiore attenzione all'aspetto nutrizionale nella prevenzione e trattamento della CKD-MBD.

La riduzione dell'assorbimento intestinale netto di fosforo rappresenta il primo provvedimento da attuare fin dalle fasi molto precoci della $\mathrm{CKD}$, al fine di prevenire o correggere le iniziali alterazioni del metabolismo calciofosforo quali l'incremento del FGF-23, l'inibizione della $1 \alpha$-idrossilasi e riduzione del calcitriolo, l'aumento della sintesi e secrezione del PTH.

In questo senso, il controllo e la riduzione dell'apporto dietetico di proteine e di fosforo, piccole dosi di calcio carbonato e la correzione di un'eventuale carenza di VitD rappresentano i provvedimenti da attuare nel nefropatico cronico.

Nel paziente in dialisi, la dieta da sola non è in grado di controllare il bilancio del fosforo per l'aumentata richiesta proteica e per problemi di aderenza, ma molto si può fare per limitare comunque il carico dietetico e quindi la positività del bilancio del fosforo.

La collaborazione del dietista è fondamentale per l'opera di educazione nutrizionale, per aiutare il paziente nella scelta dei cibi e favorire l'effettiva aderenza alle norme dietetiche, aspetto cruciale di un approccio integrato alla CKD-MBD. 